\title{
The large charge expansion and AdS/CFT
}

\author{
Anton de la Fuente ${ }^{a, b}$ and Jann Zosso ${ }^{c}$ \\ ${ }^{a}$ Kavli Institute for the Physics and Mathematics of the Universe, The University of Tokyo, \\ 5-1-5 Kashiwanoha, Kashiwa 277-8583, Japan \\ ${ }^{b}$ Theoretical Particle Physics Laboratory (LPTP), Institute of Physics, EPFL, \\ Route Cantonale, 1015 Lausanne, Switzerland \\ ${ }^{c}$ Institute for Theoretical Physics, ETH Zurich, \\ Wolfgang-Pauli-Strasse 27, 8093 Zurich, Switzerland \\ E-mail: anton.fuente@ipmu.jp, jzosso@phys.ethz.ch
}

ABSTRACT: The scaling dimensions of charged operators in conformal field theory were recently computed in a large charge expansion. We verify this expansion in a dual AdS model. Specifically, we numerically construct solitonic boson star solutions of Einstein-MaxwellScalar theory in $\mathrm{AdS}_{4}$ and find that its mass at large charge reproduces the universal form of the lowest operator dimension in the large U(1) charge sector of the dual $2+1$ dimensional CFT.

Keywords: AdS-CFT Correspondence, Conformal Field Theory, Gauge-gravity correspondence, Holography and condensed matter physics (AdS/CMT)

ARXIV EPRINT: 2005.06169 


\section{Contents}

1 Introduction $\quad 1$

2 Setup 2

3 Results 23

4 Discussion 4

\section{Introduction}

The most successful methods in quantum field theory involve a perturbation expansion in a small parameter. Recently [1-3], a new such parameter was found in conformal field theory (CFT). When two of the operators in a correlation function have a large global charge, the correlator may be computable in a large charge expansion.

Consider a $2+1$ dimensional CFT with a U(1) global symmetry. Let $\Delta$ be the scaling dimension of the lowest dimension operator of charge $Q$. The large charge expansion predicts

$$
\Delta=Q^{3 / 2} \sum_{n=0}^{\infty} \frac{c_{n}}{Q^{n}}+\text { calculable }
$$

where the $c_{n}$ are theory dependent Wilson coefficients and "calculable" can be viewed as universal quantum corrections (giving rise to precise numerical predictions $[4,5]$ ). In this note, we verify the form of (1.1) in a simple model using AdS/CFT.

The large $Q$ expansion makes use of the state-operator correspondence. Let $|Q\rangle$ be the state corresponding to the operator under consideration. By construction, $|Q\rangle$ has a non-vanishing U(1) charge density. Furthermore, it is assumed that in the infinite volume limit at fixed charge density, the U(1) symmetry is spontaneously broken. Such a state is known as a superfluid [6]. We will construct a simple model in $\mathrm{AdS}_{4}$ that is dual to a superfluid in $\mathrm{CFT}_{3}$.

By the AdS/CFT correspondence:

1. The stress tensor of the CFT is dual to a dynamical metric with asymptotically AdS boundary conditions.

2. The global $\mathrm{U}(1)$ current $J$ of the CFT is dual to a $\mathrm{U}(1)$ gauge field $A$ in AdS.

3. The non-vanishing charge density imposes the boundary condition $A^{0} \rightarrow \mu+$ $z\left\langle Q\left|J^{0}\right| Q\right\rangle$, where $z$ is the radial coordinate and $\mu$ is the chemical potential. 
Physically, the boundary condition on $A$ imposes a non-vanishing radial electric field at the boundary. Any bulk solution with such an electric field will be dual to a CFT state of large charge. However, in order to describe a superfluid, we will restrict ourselves to states that spontaneously break the global U(1) symmetry. This means that in the CFT, there is an operator $\mathcal{O}$ with $\langle\mathcal{O}\rangle \neq 0$. In AdS,

4. $\mathcal{O}$ is dual to a field $\phi$ with the boundary condition $\phi \rightarrow z^{\Delta}\langle\mathcal{O}\rangle+z^{d-\Delta} \times$ source. Since the symmetry is spontaneously broken, the source term vanishes.

A minimal model with these properties is Einstein-Maxwell-Scalar theory on asymptotically global AdS. We will numerically construct solitonic boson star solutions of this model and find that its mass at large charge reproduces (1.1).

Many aspects of Einstein-Maxwell-Scalar theory were previously studied in [7-12]. Numerics were also employed using the Poincaré patch counterpart to this model in the study of holographic superconductors [13-16].

\section{Setup}

We consider

$$
S=\frac{1}{16 \pi G_{4}} \int d^{4} x \sqrt{-G}\left[\frac{6}{L^{2}}+\mathcal{R}-\frac{1}{4} F_{a b} F^{a b}-\left(D_{a} \phi\right)^{*} D^{a} \phi+\frac{2}{L^{2}}|\phi|^{2}\right],
$$

where $G_{4}$ is the gravitational constant, $\mathcal{R}$ is the Ricci scalar, and $L$ denotes the AdS radius. ${ }^{1}$ The covariant derivative is defined as $D_{a} \phi=\partial_{a} \phi-i q A_{a} \phi$, where $q$ is the charge of the scalar field.

We are looking for static and spherically symmetric solutions and therefore choose the following ansatz for the bulk metric,

$$
d s^{2}=-g(r) b(r)^{2} d t^{2}+\frac{d r^{2}}{g(r)}+r^{2} d \theta^{2}+r^{2} \sin ^{2} \theta d \varphi^{2},
$$

where $b \rightarrow 1$ and $g \rightarrow \frac{r^{2}}{L^{2}}+1$ as $r \rightarrow \infty$ for asymptotically AdS boundary conditions.

Spherical symmetry, time-independence, radial gauge, and the restriction to electrically charged solutions allows the following ansatz for the fields,

$$
\phi=\phi(r) \quad A=a(r) d t .
$$

Moreover, the $r$-component of Maxwell equations implies that the phase of $\phi$ must be constant, allowing us to take $\phi$ to be real without loss of generality.

The equations of motion result in four coupled differential equations and the following asymptotic behaviour of the fields,

$$
\begin{aligned}
& \phi(r \rightarrow \infty)=\frac{\phi_{1}}{r}+\frac{\phi_{2}}{r^{2}}+O\left(1 / r^{3}\right) \\
& a(r \rightarrow \infty)=\mu-\frac{\rho}{r}+O\left(1 / r^{2}\right) \\
& g(r \rightarrow \infty)=r^{2}+1+\frac{\phi_{1}^{2}}{2}-\frac{m}{r}+O\left(1 / r^{2}\right) .
\end{aligned}
$$

\footnotetext{
${ }^{1}$ The equations of motion are invarant under the scaling $r \rightarrow \lambda r, t \rightarrow \lambda t, L \rightarrow \lambda L, q \rightarrow q / \lambda$ which we shall used to set $L=1$.
} 


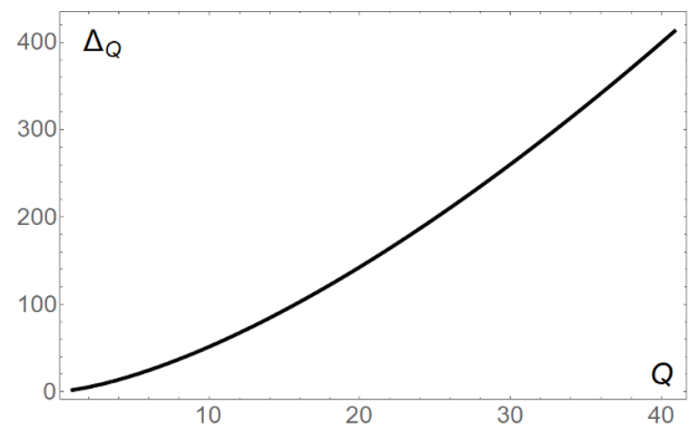

Figure 1. The numerical result of $M\left(Q_{\mathrm{AdS}}\right)=\Delta_{Q}(Q)$ along the soliton branch $q^{2}=1.4$.

To impose that the dual CFT state is a superfluid with zero entropy, the solutions we consider have no horizon, vanishing $\phi_{1}$, and non-vanishing $\mu, \rho$, and $\phi_{2}$.

The parameter $\rho$ is then related to the total charge $Q_{\text {AdS }}$ of the solution through Gauss' law, which in our convention is

$$
Q_{\mathrm{AdS}}=\frac{L^{2}}{16 \pi G_{4}} \lim _{r \rightarrow \infty} \int_{S^{2}} \star F=\frac{L^{4}}{4 G_{4}} \rho,
$$

where $S^{2}$ is a two-sphere at constant $t$ and $r$ and $\star F$ is the Hodge star dual of the field strength. Similarly, the AdS invariant boundary conditions allow us to make the link between the physical mass $M$ of the solution and the coefficient $m$ in the fall-off of the lapse function (2.6)

$$
M=\frac{L^{4}}{2 G_{4}} m .
$$

The AdS/CFT dictionary relates the boundary values $m$ and $\rho$ to the dimension $\Delta_{Q} / l$ and charge $Q$ of the CFT respectively. Following [12] we will choose units in which $2 G_{4}=1$, such that ${ }^{2}$

$$
M=m=\Delta_{Q} \quad \text { and } \quad Q_{\text {AdS }}=\frac{1}{2} \rho=Q .
$$

\section{Results}

We numerically integrated the EOMs using a shooting method and constructed horizonless solutions with non-vanishing scalar, electric, and gravitational fields. Such solutions are often referred as "boson stars" [7-11] and consists of a one parameter family for each value of the scalar charge $q$. For concreteness, we take $q^{2}=1.4$ and plot $\Delta_{Q}$ in figure 1 . Assuming the expansion (1.1), a fit of the numerical data gives the following,

$$
\Delta_{Q}^{\mathrm{fit}}=1.56369 Q^{3 / 2}+0.68411 Q^{1 / 2}-0.03174 \frac{1}{Q^{1 / 2}} .
$$

Next, we plot the difference between the numerical values of $\Delta_{Q}$ (figure 1) and the terms of $\Delta_{Q}^{\text {fit }}(3.1)$ in figures $2 \mathrm{a}$ and $2 \mathrm{~b}$. Fitting the curves $2 \mathrm{a}$ and $2 \mathrm{~b}$ with a functions

\footnotetext{
${ }^{2}$ A change to Fefferman-Graham coordinates shows that one can choose the AdS radius to coincide with the radius of the cylinder $L=l=1$.
} 


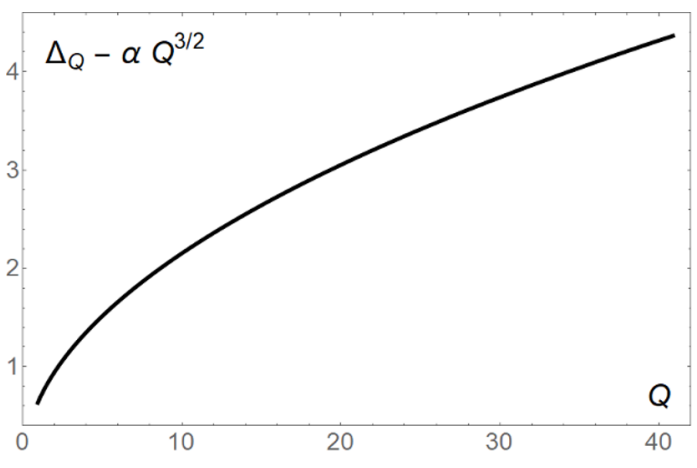

(a)

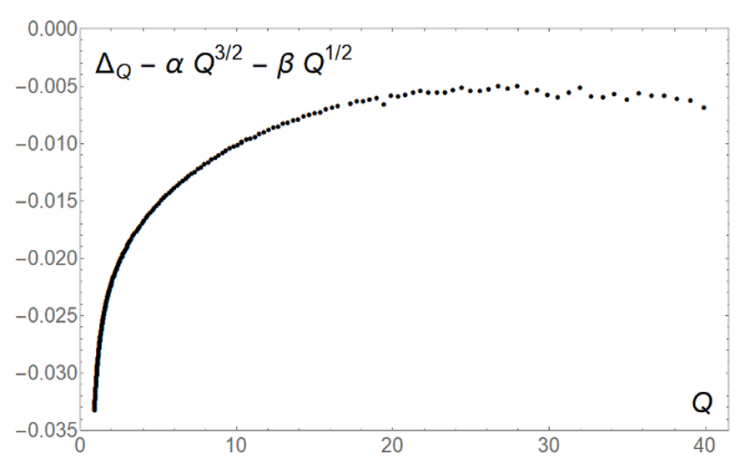

(b)

Figure 2. (a) Subtracting the first term of the fit (3.1) from the data reveals a $\sim Q^{1 / 2}$ behavior, confirming the subleading term in (1.1). (b) Subtracting also the $\beta$-term of (3.1) reveals the $\sim Q^{-1 / 2}$ behavior.

of the form $b Q^{a}$ yields $a_{b} \simeq 0.510$ and $a_{c} \simeq-0.506$ respectively, clearly showing the subleading and the second order behaviours $\sim Q^{1 / 2}$ and $\sim Q^{-1 / 2}$. Figure $2 \mathrm{~b}$ indicates that the precision of our code is starting to reach its limits at the second order. However, the agreement with (1.1) is still clearly visible. We therefore confirm the predicted universal form of the scaling dimension $\Delta_{Q}(1.1)$ of the operator of lowest dimension with charge $Q$.

\section{Discussion}

We numerically constructed boson star solutions in a simple AdS model and computed their mass as a function of their charge. We showed that the result is consistent with the prediction (1.1). Our computation was purely classical and therefore did not reproduce the "calculable" terms in (1.1). To reproduce those, we would need to include quantum fluctuations about the boson star background. Such fluctuations would be dual to the fluctuations of the goldstone boson in the CFT [17]. We note that higher order terms in the action (2.1) will presumably only change the numerical values of the coefficients $c_{n}$ in the expansion (1.1).

Apart from boson stars, the theory (2.1) also admits other charged solutions, such as black holes with scalar hair. See [18] for a phase diagram. However, such black holes do not correspond to superfluids because they have a large zero-temperature entropy. Furthermore, the Reisner-Nordstrom black hole considered in [19] does not break the U(1) symmetry and therefore does not have the correct symmetry breaking pattern of a superfluid.

In future work, we would like to compute the spectrum of small fluctuations as well as boundary correlation functions. This would allow us to further check the predictions of $[1,3]$ and to make contact with the bootstrap analysis of [20].

\section{Acknowledgments}

We thank Gabrielle Cuomo, Simeon Hellerman, Orestis Loukas, Alexander Monin, Domenico Orlando, Joao Penedones, Riccardo Rattazzi, Suzanne Reffert for discussions and conversations. The work of A.D. is partially supported by the Swiss National Science 
Foundation under contract 200020-169696, the National Center of Competence in Research SwissMAP, and the World Premier International Research Center Initiative, MEXT, Japan.

Open Access. This article is distributed under the terms of the Creative Commons Attribution License (CC-BY 4.0), which permits any use, distribution and reproduction in any medium, provided the original author(s) and source are credited.

\section{References}

[1] S. Hellerman, D. Orlando, S. Reffert and M. Watanabe, On the CFT operator spectrum at large global charge, JHEP 12 (2015) 071 [arXiv:1505.01537] [INSPIRE].

[2] L. Álvarez-Gaumé, O. Loukas, D. Orlando and S. Reffert, Compensating strong coupling with large charge, JHEP 04 (2017) 059 [arXiv: 1610.04495] [INSPIRE].

[3] A. Monin, D. Pirtskhalava, R. Rattazzi and F.K. Seibold, Semiclassics, Goldstone bosons and CFT data, JHEP 06 (2017) 011 [arXiv:1611.02912] [INSPIRE].

[4] A. De La Fuente, The large charge expansion at large N, JHEP 08 (2018) 041 [arXiv: 1805.00501] [INSPIRE].

[5] G. Badel, G. Cuomo, A. Monin and R. Rattazzi, Feynman diagrams and the large charge expansion in $3-\epsilon$ dimensions, Phys. Lett. B 802 (2020) 135202 [arXiv:1911.08505] [INSPIRE].

[6] D.T. Son, Low-energy quantum effective action for relativistic superfluids, hep-ph/0204199 [INSPIRE].

[7] P. Basu, J. Bhattacharya, S. Bhattacharyya, R. Loganayagam, S. Minwalla and V. Umesh, Small hairy black holes in global AdS spacetime, JHEP 10 (2010) 045 [arXiv:1003.3232] [INSPIRE].

[8] O.J.C. Dias, P. Figueras, S. Minwalla, P. Mitra, R. Monteiro and J.E. Santos, Hairy black holes and solitons in global $A d S_{5}, J H E P 08$ (2012) 117 [arXiv:1112.4447] [INSPIRE].

[9] Y. Brihaye, B. Hartmann and S. Tojiev, Stability of charged solitons and formation of boson stars in 5-dimensional anti-de Sitter space-time, Class. Quant. Grav. 30 (2013) 115009 [arXiv: 1301.2452] [INSPIRE].

[10] S.A. Gentle, M. Rangamani and B. Withers, A soliton menagerie in AdS, JHEP 05 (2012) 106 [arXiv: 1112.3979] [INSPIRE].

[11] S. Hu, J.T. Liu and L.A. Pando Zayas, Charged boson stars in AdS and a zero temperature phase transition, arXiv:1209.2378 [INSPIRE].

[12] Ó.J.C. Dias and R. Masachs, Hairy black holes and the endpoint of $A d S_{4}$ charged superradiance, JHEP 02 (2017) 128 [arXiv: 1610.03496] [INSPIRE].

[13] S.S. Gubser, Breaking an Abelian gauge symmetry near a black hole horizon, Phys. Rev. D 78 (2008) 065034 [arXiv:0801.2977] [INSPIRE].

[14] S.A. Hartnoll, C.P. Herzog and G.T. Horowitz, Building a holographic superconductor, Phys. Rev. Lett. 101 (2008) 031601 [arXiv: 0803.3295] [INSPIRE].

[15] S.A. Hartnoll, C.P. Herzog and G.T. Horowitz, Holographic superconductors, JHEP 12 (2008) 015 [arXiv: 0810.1563] [INSPIRE]. 
[16] G.T. Horowitz and M.M. Roberts, Zero temperature limit of holographic superconductors, JHEP 11 (2009) 015 [arXiv:0908.3677] [INSPIRE].

[17] D. Anninos, S.A. Hartnoll and N. Iqbal, Holography and the Coleman-Mermin-Wagner theorem, Phys. Rev. D 82 (2010) 066008 [arXiv: 1005.1973] [InSPIRE].

[18] P. Basu, C. Krishnan and P.N. Bala Subramanian, Phases of global AdS black holes, JHEP 06 (2016) 139 [arXiv: 1602.07211] [inSPIRE].

[19] O. Loukas, D. Orlando, S. Reffert and D. Sarkar, An AdS/EFT correspondence at large charge, Nucl. Phys. B 934 (2018) 437 [arXiv:1804.04151] [INSPIRE].

[20] D. Jafferis, B. Mukhametzhanov and A. Zhiboedov, Conformal bootstrap at large charge, JHEP 05 (2018) 043 [arXiv: 1710.11161] [INSPIRE]. 